\title{
Inclusión y diversidad, heterosexualidad y desobediencias sexo- genéricas: aportes teórico-político-epistemológicos para repensar el discurso pedagógico de la Educación Sexual Integral (ESI) Inclusion and diversity, heterosexuality, and sex/gender disobedience: theoretical-political-epistemological contributions to rethink CSE pedagogical discourse
}

\author{
ALEJANDRO D. ROJAS ${ }^{(1)}$
}

Fecha de recepción 21/04/19

Fecha de aceptación: 30/09/19

Resumen. En el discurso pedagógico de la Educación Sexual Integral (ESI), inclusión y diversidad sexual configuran un binomio conceptual relevante. Dado el uso acrítico de esta dupla, el propósito del presente trabajo es transparentar algunas de sus implicancias político-epistemológicas a través de las cuales se perpetúan privilegios y subalternidades. En relación a ello, se ha de plantear un análisis reflexivo basado en el concepto de heterosexualidad como régimen político de opresión, con la finalidad de proponer el reemplazo de una pedagogía de la inclusión de la diversidad sexual por una pedagogía celebratoria de las desobediencias sexo-genéricas.

Palabras clave. inclusión · diversidad sexual · ESI · heterosexualidad · desobediencias sexo-genéricas

\begin{abstract}
Inclusion and sexual diversity constitute a relevant conceptual binomial in the pedagogical discourse of Comprehensive Sexuality Education (CSE). Given the uncritical use of this duo, the purpose of this paper is to reveal some of its political-epistemological implications through which privileges and subalternities are perpetuated. In this regard, a reflexive analysis based on the concept of heterosexuality as a political regime of oppression should be posed with the aim of suggesting the replacement of a pedagogy based on sexual diversity inclusion by a de-heterosexualizing and celebratory pedagogy based on sex/gender disobedience.
\end{abstract}

Keywords. Inclusion - sexual diversity - CSE · heterosexuality $\cdot$ sex/gender disobedience
(1) Docente catedrático del ISP $\mathrm{N}^{\circ} 6$, del IsP $\mathrm{N}^{\circ} 60$ y del ISP $\mathrm{N}^{\circ}$ 30. Profesor en Historia (FHUC-UnL). Licenciado en Historia (FHUC-UNL). Magister en Didácticas Específicas (FHUC-UNL). Diplomado Superior en Ciencias Sociales con mención en Gestión de la Instituciones Educativas (FLACSO). Doctorando en Estudios Sociales (FHUC-UNL). Docente especialista en ESI. ale-rojas-55@hotmail.com 


\section{Introducción}

«Nuestra praxis productiva es desobedecer las normas del género y sexuales» (Preciado, 2019:309).

Diversidad e inclusión representan una dupla conceptual relevante en el discurso pedagógico de la ESI. Circula en las denominaciones de cursos, talleres y programas, en lineamientos y diseños curriculares, y en recursos didácticos para la formación docente en ESI. Solemos encontrar expresiones tales como: «respeto por la diversidad», «inclusión de los distintos», "aceptación y tolerancia de los diferentes», «atención a la diversidad», «no exclusión de las diferentes identidades sexuales», entre otras.

Como asunto de derechos humanos, de reparación histórica y de justicia curricular, «el respeto por, y la inclusión de la diversidad sexual» se ha vuelto un imperativo político-educativo insoslayable. Sin embargo, consideramos que el uso irreflexivo, acrítico y aproblemático de estas expresiones, reproducidas en el ámbito escolar, tiende a reeditar paradójicamente lugares de privilegio y subalternidad. Frente a esto, la propuesta del presente trabajo consiste en problematizar la dupla conceptual e intentar reflexionar sobre sus supuestos e implicancias político-epistemológicas, y en plantear un análisis reflexivo basado en el concepto de heterosexualidad como régimen político de opresión. Finalmente, se ha de plantear una apuesta que sustituya la pedagogía de la inclusión de la diversidad sexual por una pedagogía que se anime a festejar la disrupción de las desobediencias sexo-genéricas.

\section{Inclusión y diversidad en el discurso pedagógico de la ESI}

En primer lugar, la acción de incluir presupone que hay un adentro y un afuera, y que entre ambos espacios hay fronteras que separan a uno del otro. ¿Cuáles son las características de ese adentro y de ese afuera? ¿Quiénes están adentro y quiénes, afuera? ¿Hubo algo o alguien que haya decidido que algunos debieran estar adentro y otros afuera? En segundo lugar, supone que los de 
adentro poseen la capacidad suficiente para incluir y por tanto el poder para decidir cuándo hacerlo. ¿Cuáles son las condiciones que deben darse para que los de adentro decidan incluir a los de afuera? ¿Con qué recursos materiales y simbólicos cuentan los de adentro para operar la inclusión? ¿Cuándo se torna necesario, urgente o conveniente incluir? En tercer lugar, la acción de incluir implica que los espacios del adentro y del afuera parecen perpetuarse y que las fronteras entre ambos se resisten a desaparecer. ¿Acaso la inclusión de los excluidos no deja como saldo nuevas formas de exclusiones? Si los excluidos ya fueron incluidos, ¿por qué habría nuevos excluidos? ¿Quiénes determinan esta nueva categoría de exclusión? Si la inclusión reproduce las divisiones entre el adentro y el afuera, y con ello la continuidad de nuevos privilegios y de nuevas subalternidades, ¿cuál sería entonces el sentido político de seguir sosteniendo la categoría en cuestión?

Respecto del concepto de diversidad, en primer lugar, asistimos a una categoría polisémica, cuya semántica parece decir mucho y poco al mismo tiempo. ¿Es la diversidad un dato objetivo de la realidad, una cosa en sí misma? ¿Es algo que podamos des-cubrir corriendo velos? Y si fuese así, ¿por qué es posible reconocer otros tipos de diversidades y no las que están ligadas al sexo, el género y el deseo sexual? La diversidad, más que una realidad ontológica, es una fabricación discursiva realizada por quienes pueden crear y clasificar a la diversidad como tal.

En esta perspectiva, la diferencia se constituye, siempre, en una relación. Ella deja de ser comprendida como un dato y pasa a ser vista como una prerrogativa que es hecha a partir de un determinado lugar. Quien es representado como diferente, por otro lado, se torna indispensable para la definición y para la continua confirmación de la identidad central, ya que sirve para indicar lo que esta identidad no es o no puede ser (Louro, 2018:4).

En segundo lugar, la diversidad, como sinónimo de la otredad, remite a un todo homogéneo, que desconoce y solapa la heterogeneidad de ese gran conglomerado de cosas distintas. Es así como a la diversidad,

(...) se la destituye de su heterogeneidad y su antagonismo. La «diversidad» funciona como un término paraguas que contiene lo «otro» de la heterosexualidad, cualquier tipo de diferencia, 
con un alto grado de ambigüedad e indefinición que podría ser cualquiera y ninguna al mismo tiempo (Flores, 2015:5-6).

En este sentido, la diversidad opera como artefacto discursivo, cuya función es la de englobar lo ajeno al mundo heterosexual, pero sin hacer mención explícita del mismo como tal. En tercer lugar, «la enseñanza de la diversidad sexual» suele poner el foco en el conocimiento de las identidades como objetos de conocimiento en sí mismos, que en el mejor de los casos, no va más allá de clasificaciones y etiquetas. En aras de la visibilización, hay una necesidad de nombrar "orientaciones emergentes» a las que se les debe respeto y tolerancia. Sin embargo, esta opción de visibilizar la extrañeza minoritaria, termina convirtiendo a «los distintos» en sujetos «especiales» y frágiles a quienes se les debe aceptación y asistencia. Consideramos que

se trata de un discurso de corte liberal sobre las minorías sexuales, que tienen necesidades especiales y requieren atención específica, ayuda y tolerancia, en el marco de la igualdad. Una de las ideas que aparece de manera reiterada en este tipo de discurso es la necesidad de normalizar la homosexualidad (Trujillo, 2015:1535).

Pese a la voluntad de dar legítimo reconocimiento social a las «identidades diferentes» (¿diferentes a qué?), el discurso pedagógico de la ESI institucionaliza la separación de un «nosotros» y un «los otros». Curiosamente, cuando los "otros» adquieren visibilidad, el "nosotros» se vuelve invisible; ese "nosotros», creador de los distintos, queda oculto y por fuera de la órbita del análisis. Si hay que incluir a la diversidad, ¿cómo desenmascarar entonces a ese «nosotros» enmudecido y privilegiado que no precisa ser incluido? No es más ni menos que lo que aquí denominamos heterosexualidad.

La heterosexualidad, como lo no diferente a otra cosa, produce a los distintos; «(...) constituir una diferencia y controlarla es un acto de poder ya que es un acto esencialmente normativo. Cada cual intenta presentar al otro como diferente. Pero no todo el mundo lo consigue. Hay que ser socialmente dominante para lograrlo» (Wittig, 2006:40). Esta posición de poder hace que la exhortación a la tolerancia vaya dirigida a las personas heterosexuales, y en este sentido, es menester subrayar que las acciones de inclusión, sólo podrían tener cabida en 
tanto y en cuanto se posea la capacidad necesaria de hacerlo para con quienes ocupan un lugar de ostracismo sociosexual. Es por ello que,

asociada al diálogo y al respeto, la tolerancia parece insospechada cuando es mencionada en las políticas educativas oficiales y en los currículos. Ella se relaciona, sin embargo, con la condescendencia, la autorización, la indulgencia -actitudes que son ejercidas, casi siempre, por aquel o aquella que se percibe superior (Louro, 2018:4)

Esta superioridad misericordiosa, no transparentada como tal, hace que los enunciados pedagógicos de la tolerancia, presentes en la ESI, estén formulados desde un "nosotros heterosexual» no explicitado; tanto el sujeto enunciador como el sujeto destinatario del discurso del respeto y la inclusión, permanecen en estado tácito. He aquí que las diferencias se definen asimétricamente en relación con una hegemonía no problematizada. En este sentido, se ha de recuperar la idea según la cual, la heterosexualidad se define a sí misma sin problematizarse, se eleva como un término no marcado y privilegiado, denigrando y problematizando la homosexualidad. La homosexualidad, entonces, le da a la heterosexualidad su realidad sustancial y le permite adquirir su status por incomparecencia, como una falta de diferencia o una ausencia de anormalidad. (Halperin, 2007:65).

Esta definición político-epistemológica permite plantear que entre heterosexualidad y normalidad sexual hay una yuxtaposición conceptual, ya que dicha falta de diferencia la coloca en una posición naturalizada. Este privilegio se debe a que «la heterosexualidad ha sido históricamente construida como la sexualidad natural, legítima, respetable, legal, visible, y la masculinidad hegemónica (heterosexual) se enseña y construye en oposición al otro, o la otra, el diferente: las mujeres y los gays» (Trujillo, 2015:1531). Ello nos obliga a reconceptualizar la heterosexualidad desde otros lugares políticos y a trocar el término de diversidad sexual por el de desobediencias sexo-genéricas. 


\section{La heterosexualidad como régimen político de opresión}

La heterosexualidad no es una mera preferencia o inclinación sexual, sino más bien un régimen político basado en la opresión ejercida sobre el universo no heterosexual.

Desde el movimiento y los estudios feministas y de la disidencia sexual, la heterosexualidad no está considerada como una práctica sexual más, sino que es analizada como un régimen político que institucionaliza una forma única y legítima de vivir el deseo y los placeres, regulando de este modo los usos del cuerpo (Flores, 2016:18).

Esta institucionalización hace que la heterosexualidad sea hegemónica. ¿En qué sentido? ¿De qué manera las personas heterosexuales, además de reproducirla en forma naturalizada, gozan de sus beneficios y privilegios? Veamos algunos ejemplos.

La heterosexualidad es hegemónica, porque como algo propio de una mayoría, está omnipresente, pero no nombrada. Con pretensiones de universalidad, codifica y estructura las relaciones sociales, las instituciones y la vida cotidiana de los sujetos. "La heterosexualidad está institucionalizada como una forma particular de la práctica y de las relaciones, de la estructura de la familia y de la identidad. Se construye como una categoría coherente, natural, fija y estable; como universal y monolítica» (Richardson, 1996:124). Es hegemónica porque las personas heterosexuales no necesitan confesar su heterosexualidad, no están obligadas a salir del closet para liberarse y no son interrogadas acerca de los episodios desencadenantes de su «inclinación heterosexual» porque allí, en ello, no hay confusión; los vínculos heterosexuales están naturalizados.

Y por mucho que se haya admitido en estos últimos años que no hay naturaleza, que todo es cultura, sigue habiendo en el seno de esta cultura un núcleo de naturaleza que resiste al examen, una relación excluida de lo social en el análisis y que reviste un carácter de ineluctabilidad en la cultura como en la naturaleza: es la relación heterosexual (Wittig, 2006:38).

Esta naturalización de la relación heterosexual hace que las personas heterosexuales no sufran el estigma social. No son asesinadas, lastimadas, ni perse- 
guidas por haberse declarado en público como tales, porque no es necesario verbalizarlo. Las personas heterosexuales habitan el espacio público en forma privilegiada, porque por demostrar cariño en una plaza, no son censuradas, expulsadas y detenidas por las fuerzas policiales, ya que ello no podría ser considerado un escándalo público. De ahí que,

La distinción privado/público es, entonces, una noción sexualizada: tiene un significado diferente dependiendo de si uno lo está aplicando a un contexto heterosexual u homosexual. Para las lesbianas y los gais, lo privado se ha institucionalizado como la frontera de la tolerancia social, como el lugar donde se permite vivir relativamente con seguridad mientras no procuren ocupar el espacio público (Richardson, 1996:146).

Ahora bien, ¿cómo opera el régimen heterosexual en las instituciones escolares?, ¿cuáles son los discursos y las prácticas heterosexualizantes que aún persisten con una fuerza naturalizada extraordinaria? Veamos algunos ejemplos ilustrativos.

En la educación inicial, funcionan prácticas heterosexistas, tales como: la formación de filas basadas en el sexo en forma inconfundible, la separación arbitraria de los espacios lúdicos y los juguetes para niños y niñas, la presencia de los colores celeste y rosa asignados a varones y a mujeres respectivamente, las prohibiciones a los niños cuando desean jugar con muñecas y colocarse un vestido. En la educación primaria, las prácticas heterosexualizantes se manifiestan cuando una maestra cita a la madre de una alumna, porque ésta sólo juega en los recreos con los varones; cuando a una alumna se le llama la atención, indicándole cómo debería comportarse "una señorita», resguardando así su feminidad; cuando se presume que la familia de un alumno está constituida por un padre y una madre; cuando está permitido que en los actos escolares las niñas se vistan de varones para representar figuras masculinas, pero no cuando se trata de los varones vestidos de mujeres para representar figuras femeninas; un varón tra-vestido es inadmisible. En la educación secundaria, la heterosexualidad opera cuando un preceptor recomienda a un alumno que no hable demasiado de su elección homosexual para no ser discriminado por sus compañeros; cuando nos encontramos con insultos homo/lesbo/transodiantes plasmados en grafitis en los baños; cuando un alumno disgustado no asiste a las 
clases de Educación Física, porque éstas se reducen a la práctica de deportes tales como el fútbol; cuando se presume la heterosexualidad de una docente; cuando en las prácticas institucionales se perpetúa la elección de «la reina de la primavera» y la del «mariposón» en el día del estudiante; cuando un adolescente que desea cambiar de género se ve obligado a abandonar el cursado regular.

\section{De la inclusión de la diversidad a la celebración de las desobediencias}

Las nociones ensayadas en torno al concepto de heterosexualidad como régimen político de opresión, que subalterniza al mundo no heterosexual, devienen incompatibles con el discurso pedagógico de la inclusión y la diversidad. ¿Incluir implica incorporar la diversidad como otredad al «nosotros heterosexual»? ¿Incluir trae como consecuencia nuevos disciplinamientos? ¿El espacio «del adentro» quedaría conformado por una mayoría heteronormal y una minoría diversa? Si hay «un adentro» así configurado, ¿quiénes están ahora afuera como saldo de las nuevas inclusiones? ¿Acaso otorgar a la heterosexualidad, el rol de incluir no reedita subrepticiamente relaciones de poder? La retórica de la diversidad «(...) nos instala en una epistemología neoliberal y colonial, en la que la compasión, la tolerancia, el respeto, la simpatía, constituyen fórmulas medulares de su prédica victimizante y paternalista» (Flores, 2015:5). ¿Acaso este poder paternal, no supone poner a los distintos en una posición de minoridad, de minusvalía y de deuda para con el mundo heterosexual?

En función de estas reflexiones, proponemos que el discurso pedagógico de la ESI abra la posibilidad de reemplazar el concepto de diversidad sexual por el de desobediencias sexo-genéricas. En primer lugar, esta sustitución conceptual posa la mirada en una idea políticamente más potente, puesto que des-cubre y denuncia la hegemonía heterosexual y los procesos de subalternización sexo-genérica, y habilita pensar en la oportunidad de fisurar los discursos del orden heteronaturalizado en términos de oposición y de lucha, de resistencia y de conflicto. En segundo lugar, el reemplazo de las categorías en cuestión permite repensar política y positivamente a las personas no heterosexuales como desertoras, traidoras, desviadas y fugitivas. Las personas desobedientes 
al orden heterosexual, desertan del ejército militante de la heterosexualidad como norma; traicionan el sexo, el género y el deseo sexual del mundo heterosexual; se desvían de los senderos esperados y deseados de los despotismos heterosexuales; fugan de la heterosexualidad compulsiva, de sus dispositivos familiares y escolares, de su insistencia en modelar deseos y cuerpos, placeres y estilos de vida. Dado este carácter de desobediencia debida, que se niega a ser asimilado al «nosotros inclusivo heterosexual», ¿cuál sería entonces el sentido de persistir obstinadamente en las ideas tolerar e incluir desde la piedad y la bondad heterosexuales a quienes no lo desean? El epígrafe seleccionado para el presente ensayo intenta dar cuenta de ello.

Los discursos pedagógicos han domesticado históricamente las conductas, los cuerpos y los deseos de los sujetos que habitan el escenario educativo. Su prédica constante de la obediencia, deviene anacrónica respecto del término político-sexual de la desobediencia que aquí proponemos. La apuesta, pues, consiste en que la pedagogía de la ESI vaya más allá de las máximas de la tolerancia y la inclusión. ¿Por qué no festejar la disrupción de las desobediencias sexo-genéricas?, ¿por qué no empezar a celebrar a quienes se desobedecen al régimen?, ¿por qué no abandonar las connotaciones negativas de los epítetos de desviados y anormales?, ¿por qué no alegrarse con quienes no responden al control heterosexual? ¿Acaso no es el momento propicio para abandonar la mezquindad heterosexual de incluir a quienes como divergentes luchan por ampliar horizontes de lo posible y lo deseable, desdibujando de esta manera los límites del «adentro» y del "afuera»? Des-heterosexualizar(nos) e ir hacia una pedagogía celebratoria...

\section{Consideraciones finales}

Hemos intentado problematizar uno de los binomios centrales del discurso pedagógico de la ESI, tal como lo es la dupla conceptual de inclusión-diversidad, con la finalidad de reflexionar críticamente sobre algunas de sus implicancias político-epistemológicas, mediante las cuales se reproducen posiciones de privilegio y subalternidad. En relación a ello, reconceptualizamos la heterosexualidad 
como régimen político de opresión y propusimos la sustitución del concepto de diversidad sexual por el de desobediencias sexo-genéricas, cuya definición es incompatible y anacrónica respecto de la pedagogía de la inclusión. Finalmente, debido a esta incongruencia, hemos planteado como apuesta una pedagogía que se abra al festejo de las desobediencias.

Esta pedagogía de la celebración supone pensar en la posibilidad de abolir la institucionalización de la heterosexualidad, de claudicar en la acción de incluir y ampliar el espectro de lo posible y de lo deseable, de desarticular los artefactos materiales y simbólicos de los que se vale la heterosexualidad para perpetuarse como mandato, de estimular la deslegitimación de la heterosexualidad como destino inexorable y de fisurar la ortodoxia de una presunta naturaleza heterosexual. Irreverentes del canon heterosexual, las desobediencias dan la espalda al sexo asignado al nacer, subvierten el orden (hetero) sexual de las cosas en sus cuerpos y sus deseos; desacatan las leyes decretadas por el régimen. En ellas, el proyecto heteronormativo ha fracasado, porque representan las líneas de fuga y los efectos que el poder heterosexual no pudo (porque no puede) controlar. Producidas por el régimen político heterosexual como identidades abyectas, las desobediencias sexo-genéricas reniegan de la inclusión al mundo heteronormado.

Superada la conceptualización de la heterosexualidad como mera inclinación sexual hacia el sexo opuesto (iopuesto a qué?), y habiendo propuesto el reemplazo de diversidad por desobediencia, apostamos en este sentido a que la política pedagógica de la tolerancia, el respeto y la inclusión indulgente logre transformarse en una pedagogía celebratoria de la disrupción de las desobediencias sexo-genéricas. 


\section{Referencias bibliográficas}

Flores, V. (2015). ESI: Esa Sexualidad Ingobernable. EI reto de des-heterosexualizar la pedagogía. Presentado en Degenerando Buenos Aires. III Jornadas Interdisciplinarias de Géneros y Disidencia Sexual organizado por Cauce UBA y Desde el Fuego, Buenos Aires.

Flores, V. (2016). Afectos, pedagogías, infancias y heteronormatividad. Reflexiones sobre el daño. En Editor, Britzman, D., Flores, V. \& Hooks, Bell (Ed.) Pedagogías transgresoras (pp. 13-30). Córdoba: Bocavulvaria.

Halperin, D. (2007). San Foucault. Para una hagiografía gay. Buenos Aires: El cuenco de plata.

Louro, G. L. (2018). Currículo, género y sexualidad. Lo normal, lo diferente y lo excéntrico. Descentrada, volumen (3). Recuperado de https://www.descentrada. fahce.unlp.edu.ar/article/view/DESe065
Preciado, P. B. (2019). Un apartamento en Urano: crónicas del cruce. Barcelona: Anagrama.

Richardson, D. (1996). Theorising Heterosexuality: Telling It Straight. Buckingham: Open University Press. Trujillo, G. (2015). Pensar desde otro lugar, pensar lo impensable: hacia una pedagogía queer. Educ. Pesqui., São Paulo, volumen (41), 1527-1540. Recuperado de http:// dx.doi.org/10.1590/S1517-9702201508142550

Wittig, M. (2006). El pensamiento heterosexual y otros ensayos. Barcelona: Egales. 